\title{
Development of derivative spectrophotometric method for simultaneous determination of pyrazinamide and rifampicin in cubosome formulation
}

\author{
Morgana Souza Marques ${ }^{\mathrm{a}}$, Fernando Dal Pont Morisso $^{\mathrm{b}}$, Fernanda Poletto ${ }^{\mathrm{c}}$, Irene Clemes Külkamp \\ Guerreiro $^{\mathrm{a}^{*}}$
}

\begin{abstract}
${ }^{a}$ Pós-Graduação em Ciências Farmacêuticas, Universidade Federal do Rio Grande do Sul, 90610-000, Porto Alegre, RS, Brazil; ' ${ }^{P o ́ s-G r a d u a c ̧ a ̃ o ~ e m ~ T e c n o l o g i a ~ d e ~ M a t e r i a i s ~ e ~ P r o c e s s o s ~ I n d u s t r i a i s, ~ U n i v e r s i d a d e ~ F e e v a l e, ~ 93525-075, ~}$

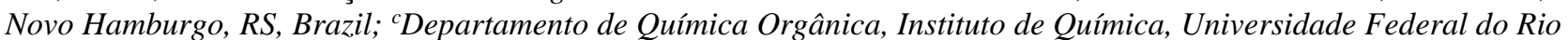
Grande do Sul, 91501-970, Porto Alegre, RS, Brazil *Corresponding author: irene@ufrgs.br
\end{abstract}

The ultraviolet spectrophotometry analysis for quantitative assay of drugs is a method accurate, sensitive, selective and reproductive with the advantage of being a simple and less expensive method. In this study, a derivative ultraviolet spectrophotometric method was developed for simultaneous determination of pyrazinamide (PYZ) and rifampicin (RIF). The spectrophotometric method was evaluated according to validation guidelines for specificity, linearity, limits of detection and quantification, precision, accuracy and robustness. The first-derivative spectra were obtained and by the zerocrossing point, the wavelength $247 \mathrm{~nm}$ and $365 \mathrm{~nm}$ were selected for PYZ and RIF quantification, respectively. No interference from cubosome excipients was detected in the proposed method. The results demonstrated linearity in a range of $4.0-12.0 \mu \mathrm{g} / \mathrm{mL}$ with an adequate correlation coefficient for both drugs. The intra and inter-day precision results (RSD $<5 \%$ ) indicated the reproducibility of the method. The accuracy data showed satisfactory results (RSD < 5\%) from recovery test. In addition, the robustness results showed that the PYZ and RIF content were unaffected by the solvent alteration of methanol to methanol:water $(99: 1, \mathrm{v} / \mathrm{v})$. The derivative ultraviolet spectrophotometric method proved to be an excellent strategy for simultaneous determination of PYZ and RIF.

Keywords: Pyrazinamide, Rifampicin, UV spectrophotometry, derivative spectrophotometry.

https://doi.org/10.22456/2527-2616.111454

\section{Introduction}

World Health Organization recognizes four drugs for tuberculosis diseases: pyrazinamide (PYZ), rifampicin (RIF), isoniazid and ethambutol (1). Pyrazinamide (pyrazine-2-carboxamide; Figure 1) is classified as a crystalline white or almost white powder, slightly soluble in water and ethanol (2). This drug is an analog of nicotinamide with bactericidal property (3). The mechanism of action of PYZ is unknown and experimental evidence suggests that bactericidal action is dependent of the pyrazinamidase enzyme from Mycobacterium tuberculosis (4). Once PYZ is converted into pyrazinoic acid by bacterial pyrazinamidase, the high concentrations of pyrazinoic acid in the bacterial cytoplasm decrease the intracellular $\mathrm{pH}$ causing inactivation of vital enzymes, resulting in the death of the bacillus (5).

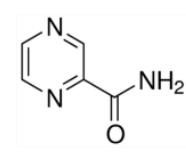

Pyrazinamide

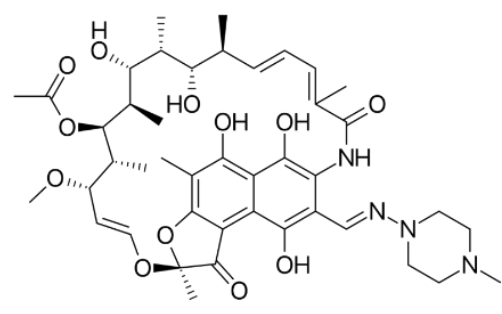

Rifampicin
Figure 1. Chemical structure of pyrazinamide and rifampicin.

\section{RIF}

((7S,9E,11S,12R,13S,14R,15R,16R,17S,18S,19E,21Z)-

$2,15,17,27,29$-pentahydroxy-11-methoxy-

3,7,12,14,16,18,22-heptamethyl-26-[(1E)-[(4-

methylpiperazin-1-yl)imino]methyl]-6,23-dioxo-8,30-

dioxa-24-azatetracyclo[23.3.1.1 $\left.{ }^{4},{ }^{7} .0^{5},{ }^{28}\right]$ triaconta-

$1(29), 2,4,9,19,21,25,27$-octaen-13-yl acetate; Figure 1) is a red to orange odorless powder, very slightly soluble in water and ethanol; and soluble in methanol (2). RIF is a semi-synthetic antibiotic derived from rifamycin $B$, produced by strains of Amycolatopsis rifamycinica (6). It presents a broad spectrum bactericidal action, inhibiting the activity of the DNA-dependent RNA polymerase enzyme, which prevents the synthesis of messenger RNA (mRNA) and protein by the bacillus, causing cell death (7).

In order to prevent the emergence of bacterial resistance, PYZ and RIF are used in combination $(1,3)$. In addition to the pharmacotechnical challenges for the preparation of formulations containing this association, it is necessary to develop new analytical methods for the simultaneous determination of drugs. Several published studies used different analytical techniques for the determination of antituberculosis drugs alone and in combination, including High Performance Liquid Chromatography (HPLC) with UV detector $(8,9)$, High Performance Thin Layer Chromatography (HPTLC) (10), Ultra-high Performance Liquid Chromatography (UHPLC) with UV detector $(11,12)$, Fourier Transform Infrared (FTIR) spectroscopy in combination with multivariate calibration of partial least square (13) and UV spectrophotometry (14). Due to the absence of an analytical method for the simultaneous 
determination of PYZ and RIF by derivative spectrophotometric method and considering the importance of analytical methods for the simultaneous determination of drugs, this study aims to develop and validate a simple and rapid derivative spectrophotometric method for the determination of PYZ and RIF in cubosome formulation.

\section{Experimental section}

\section{Materials}

The PYZ and RIF (pharmaceutical grade) were gently donated by Fundação para o Remédio Popular (Brazil) and Farmanguinhos (Brazil), respectively. The phytantriol (3,7,11,15-tetramethyl-1,2,3-hexadecanetriol, 96.9\%) and poloxamer 407 (PEO98-PPO67-PEO98, MW 12,500 $\mathrm{g} / \mathrm{mol}$ ) were purchased from Alianza (Brazil) and Via Farma (Brazil), respectively. Methanol analytical grade was supplied from J.T. Barker and was used to prepare all solutions for analysis.

\section{Preliminary tests}

The validation of the PYZ and RIF quantification by ultraviolet spectrophotometry method started with preliminary tests (data not showed). Different solvents were tested, such as acetonitrile, phosphate buffer, water, ethanol and methanol. The methanol was selected since it had a great capability to dissolve the phytantriol, the main excipient of the cubosomes.

\section{Instrumental}

The PYZ and RIF were quantified by spectrophotometric method carried out on LAMBDA 265 UV/Vis Spectrophotometer (PerkinElmer, USA) connected to UV LAB 4.0.0 software. The absorbance was determined within the range of 200 to $400 \mathrm{~nm}$ using matched $1 \mathrm{~cm}$ quartz cells. Measurements were performed using a standard solution of PYZ and RIF in methanol in order to obtain first-derivative spectra using the zero crossing point.

\section{Method validation}

The spectrophotometric method for simultaneous determination of PYZ and RIF was validated for specificity, linearity, limits of detection (LOD) and quantification (LOQ), precision, accuracy and robustness according to the International Conference on Harmonization (ICH) guidelines Q2 (R1) for the validation of analytical methods (15) and to RDC n ${ }^{\circ} 166$ of July 24, 2017 (16).

\section{Preparation of standard solutions}

For the preparation of the standard solution, PYZ (5 mg) and RIF (5 mg) were dissolved, separately, in methanol (10 $\mathrm{mL}$ ) and sonicated for 5 minutes.

\section{Preparation of unloaded cubosomes}

Cubosomes were prepared as described previously (17) with some modifications. Phytantriol $(750 \mathrm{mg})$, poloxamer 407 (187.5 $\mathrm{mg})$ and deionized water $(30 \mathrm{~mL})$ were homogenized for $25 \mathrm{~min}$ by a $500 \mathrm{~W}$ ultrasonic processor (Ecosonics, Brazil) at $99 \%$ of amplitude and with a $13 \mathrm{~mm}$ diameter probe. The final volume was corrected to $30 \mathrm{~mL}$ with deionized water.

\section{Specificity}

Specificity was evaluated by analyzing solutions of unloaded cubosomes. The system response was determined through the presence of overlaps of unloaded cubosomes with the PYZ and RIF response.

\section{Linearity}

The linearity experiments were conducted from a standard solution of PYZ and RIF. Aliquots of standard solution were diluted in methanol to obtain the final concentrations of $4.0,6.0,8.0,10.0$ and $12.0 \mu \mathrm{g} / \mathrm{mL}$ of PYZ and RIF. All solutions were prepared in triplicate and analyzed on three different days. Calibration curves (concentration vs absorbance) were plotted, and the equation of the line was determined through the linear regression, using the method of least squares. The analysis of variance (ANOVA) was also calculated for the statistical parameters. The linearity was expressed as the correlation coefficients.

\section{Limits of detection and quantitation}

The LOD and LOQ were estimated using the standard deviation of the value of the intercept with the $\mathrm{Y}$ axis and slope of three calibration curves. The LOD and LOQ were evaluated based on the previously constructed calibration curve.

\section{Precision}

For repeatability (intra-day precision) experiment, six solutions containing a combination of PYZ and RIF at central point of the standard curves (concentration of 8.0 $\mu \mathrm{g} / \mathrm{mL}$ of each drug) were evaluated on the same day under the same experimental conditions. The intermediate precision (inter-day precision) of the method was assessed with PYZ $(8.0 \mu \mathrm{g} / \mathrm{mL})$ and RIF $(8.0 \mu \mathrm{g} / \mathrm{mL})$ solution and performed on three different days. The experimental results were expressed as the relative standard deviations (RSD).

\section{Accuracy}

The accuracy experiments were determined by applying the method to quantify PYZ and RIF in the presence of the unloaded cubosome. Unloaded cubosomes were spiked with amount of PYZ and RIF at concentrations of 4.0, 8.0 and $12.0 \mu \mathrm{g} / \mathrm{mL}$ of each drug. The analyses were performed in triplicates. Accuracy was expressed as percentage of 
PYZ and RIF recovered in the lowest, intermediate and highest concentrations.

\section{Robustness}

The robustness was determined by small variation in the established analytical condition. Samples of PYZ (8.0 $\mu \mathrm{g} / \mathrm{mL})$ and RIF $(8.0 \mu \mathrm{g} / \mathrm{mL})$ prepared with methanol:water $(99: 1$, v/v) were utilized to conducted the test. The experimental results were expressed as the RSD values.

\section{Results and Discussion}

\section{Validation of the quantification method}

The spectrophotometry analysis for determination of drugs is a method accurate, sensitive, selective, reproductive and proved to be a less expensive alternative when compared to the HPLC method. However, analysis of drug mixtures by ultraviolet-visible spectrophotometry is often compromised due to the overlap of the electronic transition bands. As seen in Figure 2, the zero-order spectra of PYZ and RIF showed an overlapping, making the detection and quantification of drugs unfeasible. The zero-order spectra of PYZ and RIF were processed to obtain first-derivative spectra. By overlapping spectra of first-derivatives, it is possible to individualize the constituents and even eliminate the interference of one component on the other. The spectra of the first-derivative of PYZ and RIF were superimposed and showed that at wavelength $247 \mathrm{~nm}$ there is the annulment of the spectrum of the drug RIF as well as at wavelength $365 \mathrm{~nm}$, the annulment of PYZ occurs (Figure 3).

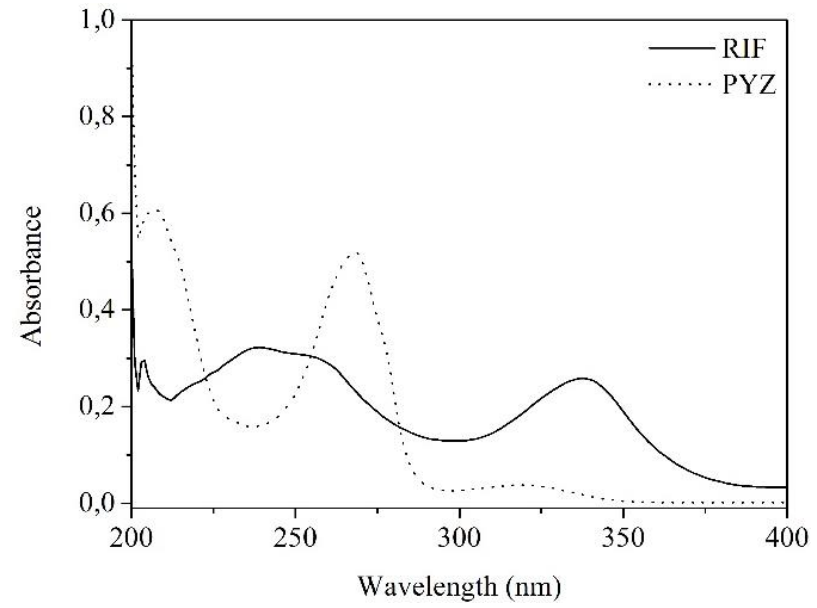

Figure 2. Zero-order absorption spectra of PYZ $(8.0 \mu \mathrm{g} / \mathrm{mL})$ and RIF $(8.0 \mu \mathrm{g} / \mathrm{mL})$ obtained in methanol.

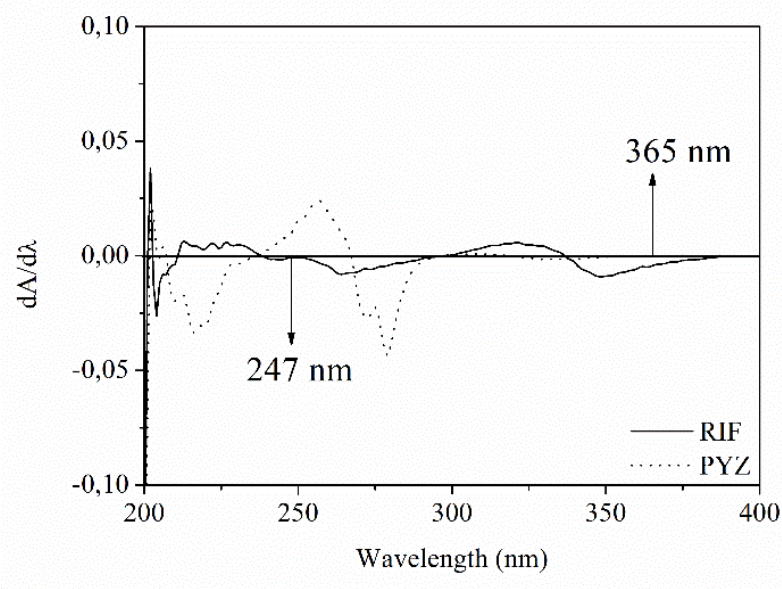

Figure 3. Absorption spectra of the first-derivative spectra of PYZ $(8.0 \mu \mathrm{g} / \mathrm{mL})$ and $\mathrm{RIF}(8.0 \mu \mathrm{g} / \mathrm{mL})$ obtained in methanol.

\section{Specificity}

The interference of the unloaded cubosome at the PYZ and RIF measurement was demonstrated in the specificity test. As clearly seen in the first-derivative spectrum of unloaded cubosome (Figure 4), a minimum absorption of 0.0002 at $247 \mathrm{~nm}$ and 0.0001 at $365 \mathrm{~nm}$ was observed. These results are in agreement with previous studies $(18,19)$, which observed that the first-derivative spectrophotometric method was able to eliminate the interference from excipients. The proposed analytical method is able to quantify these specific drugs in the presence of a complex matrix, such cubosomes, that contain the inputs phytantriol and poloxamer 407 .

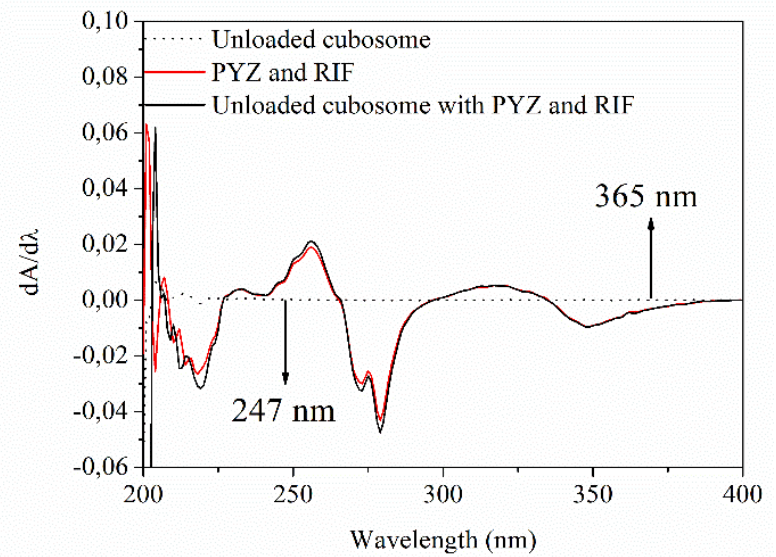

Figure 4. Absorption spectra of the first-derivative of unloaded cubosome, standard solution of PYZ and RIF and unloaded cubosome with standard solution of PYZ and RIF.

\section{Linearity}

The results from linearity test are summarized in Table 1 . The least square regression showed excellent correlation between the PYZ concentration and the peak amplitude $(247 \mathrm{~nm})$ in the range of 4.0 and $12.0 \mu \mathrm{g} / \mathrm{mL}$ with $\mathrm{r}$ value of 0.999 . Similarly correlation was observed for the RIF concentration and peak amplitude at $365 \mathrm{~nm}(\mathrm{r}=0.998)$. Statistical analysis by ANOVA confirmed a linear 
regression for PYZ $\left(\mathrm{F}_{\text {calculated }}=608.19>\mathrm{F}_{\text {tabulated }}=4.96\right.$; $\mathrm{p}=0.05)$ and $\operatorname{RIF}\left(\mathrm{F}_{\text {calculated }}=573.97>\mathrm{F}_{\text {tabulated }}=4.96\right.$; $\mathrm{p}=0.05$ ) and also shows that there is no deviation from linearity for PYZ $\left(\mathrm{F}_{\text {calculated }}=0.20<\mathrm{F}_{\text {tabulated }}=3.71 ; \mathrm{p}=0.05\right)$ and $\operatorname{RIF}\left(\mathrm{F}_{\text {calculated }}=3.65<\mathrm{F}_{\text {tabulated }}=3.71 ; \mathrm{p}=0.05\right)$.

Table 1. Linearity results obtained from standard curve of firstderivative spectrophotometric method for pyrazinamide and rifampicin quantification.

\begin{tabular}{|c|c|c|}
\hline \multirow{2}{*}{ Parameter } & \multicolumn{2}{|c|}{ Results } \\
\hline & PYZ & RIF \\
\hline Linear range & $4.0-12.0 \mu \mathrm{g} / \mathrm{mL}$ & $4.0-12.0 \mu \mathrm{g} / \mathrm{mL}$ \\
\hline $\begin{array}{l}\text { Regression } \\
\text { equation }\end{array}$ & $y=0.001 x-0.0007$ & $y=-0.0006 x+0.0001$ \\
\hline $\begin{array}{l}\text { Correlation } \\
\text { coefficient (r) }\end{array}$ & 0.999 & 0.998 \\
\hline
\end{tabular}

\section{Limits of detection and quantitation}

The calculated LOD for PYZ and RIF was $0.87 \mu \mathrm{g} / \mathrm{mL}$ and $0.82 \mu \mathrm{g} / \mathrm{mL}$, respectively, which demonstrated a great sensitivity to detect the drugs. The LOQ was estimated at $2.89 \mu \mathrm{g} / \mathrm{mL}$ for PYZ and $2.73 \mu \mathrm{g} / \mathrm{mL}$ for RIF. The results show that the proposed method allowed detection and quantification of low drug concentration, indicating that the method is sensitive enough for both drugs.

\section{Precision}

The repeatability (intra-day precision) and intermediate precision (inter-day precision) data expressed as RSD are showed in Table 2. The first-derivative spectrophotometric method for simultaneous quantification of PYZ and RIF demonstrated an adequate repeatability. The RSD for both drugs were less than $5.0 \%$, which are in accordance with the guidelines. Similar results were observed in intermediate precision (inter-day precision) with acceptable RSD range $(\leq 5.0 \%)$ which represents an agreement between the results obtained when performing the analysis in the same laboratory but on different days.

Table 2. Repeatability and intermediate precision values of firstderivative spectrophotometric method for pyrazinamide and rifampicin quantification.

\begin{tabular}{|c|c|c|c|c|}
\hline Parameter & $\begin{array}{c}\text { PYZ } \pm \\
\text { SD } \\
(\mu \mathrm{g} / \mathrm{mL})\end{array}$ & $\begin{array}{l}\text { RSD } \\
(\%)\end{array}$ & $\begin{array}{c}\text { RIF } \pm \\
\text { SD } \\
(\mu \mathrm{g} / \mathrm{mL})\end{array}$ & $\begin{array}{l}\text { RSD } \\
(\%)\end{array}$ \\
\hline Repeatability $^{\mathrm{a}}$ & $\begin{array}{c}7.86 \pm \\
0.18 \\
\end{array}$ & 2.25 & $\begin{array}{l}7.67 \pm \\
0.15\end{array}$ & 2.00 \\
\hline \multicolumn{5}{|l|}{$\begin{array}{c}\text { Intermediate } \\
\text { precision }\end{array}$} \\
\hline Day $1^{\text {b }}$ & $\begin{array}{c}8.012 \pm \\
0.17\end{array}$ & 2.09 & $\begin{array}{l}7.09 \pm \\
0.23\end{array}$ & 3.21 \\
\hline Day $2^{b}$ & $\begin{array}{c}7.79 \pm \\
0.17\end{array}$ & 2.19 & $\begin{array}{c}7.69 \pm \\
0.27\end{array}$ & 3.48 \\
\hline Day $3^{\text {b }}$ & $\begin{array}{c}7.80 \pm \\
0.13\end{array}$ & 1.70 & $\begin{array}{l}7.38 \pm \\
0.23 \\
\end{array}$ & 3.17 \\
\hline
\end{tabular}

${ }^{\mathrm{a}}$ Mean of six determinations; ${ }^{\mathrm{b}}$ Mean of three determinations.

\section{Accuracy}

The method accuracy was studied at three different levels (low, medium and high concentration) and by adding unloaded cubosomes. The drugs recovery experiments were carried, and the results are shown at Table 3.

Table 3. Recovery results of pyrazinamide and rifampicin in the presence of unloaded cubosomes analyzed by derivative spectrophotometric method.

\begin{tabular}{cccccc}
\hline \multirow{2}{*}{ Level } & Drugs & \multicolumn{4}{c}{ Concentration $(\boldsymbol{\mu g} / \mathbf{m L})$} \\
\cline { 3 - 6 } & & $\begin{array}{c}\text { Amount } \\
\text { added }\end{array}$ & $\begin{array}{c}\text { Amount } \\
\text { found }\end{array}$ & $\begin{array}{c}\text { Recovery } \\
(\%)\end{array}$ & $\begin{array}{c}\text { RSD } \\
(\%)\end{array}$ \\
\hline \multirow{2}{*}{1} & $\mathrm{PYZ}^{\mathrm{q}}$ & 4.00 & 4.67 & 116.67 & 2.47 \\
& $\mathrm{RIF}^{\mathrm{a}}$ & 4.00 & 3.78 & 94.44 & 2.55 \\
2 & $\mathrm{PYZ}^{\mathrm{a}}$ & 8.00 & 8.21 & 102.63 & 3.40 \\
& $\mathrm{RIF}^{\mathrm{a}}$ & 8.00 & 7.72 & 96.53 & 2.49 \\
\multirow{2}{*}{3} & $\mathrm{PYZ}^{\mathrm{a}}$ & 12.00 & 12.40 & 103.33 & 1.40 \\
& $\mathrm{RIF}^{\mathrm{a}}$ & 12.00 & 11.73 & 97.73 & 4.45 \\
\hline \multirow{2}{*}{ a Mean of three determinations. }
\end{tabular}

The derivative spectrophotometric method was considered accurate for the proposed drugs, presenting a satisfactory RSD value between $1.40 \%$ and $4.45 \%$.

\section{Robustness}

The results of the robustness are summarized in Table 4. The PYZ and RIF content were unaffected by the alteration of the methanol concentration used in the sample preparation, with RSD values lower than $5 \%$ for both drugs. This results demonstrate that little variation in the proportion of methanol:water used in the preparation of the samples did not influence the drug recovery.

Table 4. Effects of the variation of analytical parameters in the pyrazinamide and rifampicin content for the robustness test.

\begin{tabular}{cccc} 
Drugs & $\begin{array}{c}\text { Mean } \\
\text { concentration } \\
\mathbf{\mathbf { S D }}(\boldsymbol{\mu g} / \mathbf{m L})\end{array}$ & $\begin{array}{c}\text { Mean recover } \\
(\boldsymbol{\%})\end{array}$ & $\begin{array}{c}\text { RSD } \\
(\boldsymbol{\%})\end{array}$ \\
\hline $\mathrm{PYZ}^{\mathrm{a}}$ & $8.12 \pm 0.11$ & 101.52 & 1.35 \\
$\mathrm{RIF}^{\mathrm{a}}$ & $7.75 \pm 0.17$ & 96.84 & 2.21 \\
\hline
\end{tabular}

${ }^{\mathrm{a} M e a n}$ of six determinations.

\section{Conclusions}

A first-derivative spectrometric method was developed and validated for simultaneous determination of PYZ and RIF in cubosome formulation. The results of validation showed that the analytical method demonstrating to be simple, rapid, sensitive, precise and accurate.

\section{Acknowledgments}

The authors would like to thank the Conselho Nacional de Desenvolvimento Científico e Tecnológico (CNPq) for financial support (M.S.M. Doctorate Scholarship - 141883 ( 2018-9), Fundação de Amparo à Pesquisa do Estado do Rio Grande do Sul (19/2551-0001824-0), Feevale University for access to UV-Vis spectrophotometer equipment, Fundação para o Remédio Popular and Farmanguinhos for the supply of drugs and Dr. Juliana Emanuelli for the support in the statistical analysis. 


\section{Conflict of interest}

The authors declare no conflicts of interest.

\section{References}

1. WHO. Global tuberculosis report. 2017.

2. Farmacopeia Brasileira. 6 edição. 2019.

3. Donald PR, Maritz JS, Diacon AH. Pyrazinamide pharmacokinetics and efficacy in adults and children. Vol. 92, Tuberculosis. Churchill Livingstone; 2012. p. $1-8$.

4. Zhang Y, Mitchison D. The curious characteristics of pyrazinamide: a review. nternational Union Against Tuberc Lung Dis. 2003;7(1):6-21.

5. Zhang Y, Shi W, Zhang W, Mitchison D. Mechanisms of pyrazinamide action and resistance. Microbiol Spectr. 2013;2(4):1-12.

6. Bala S, Khanna R, Dadhwal M, Prabagaran SR, Shivaji S, Cullum J, et al. Reclassification of Amycolatopsis mediterranei DSM 46095 as Amycolatopsis rifamycinica sp. nov. Int $\mathbf{J}$ Syst Evol Microbiol. 2004;54:1145-9.

7. Floss HG, Yu T-W. Rifamycin - Mode of Action, Resistance, and Biosynthesis. Chem Rev. 2005 Feb;105(2):621-32.

8. Chellini PR, Lages EB, Franco PHC, Nogueira FHA, César IC, Pianetti GA. Development and Validation of an HPLC Method for Simultaneous Determination of Rifampicin, Isoniazid, Pyrazinamide, and Ethambutol Hydrochloride in Pharmaceutical Formulations. J AOAC Int. 2015 Sep 1;98(5):1234-9.

9. Farmacopeia Americana. USP 43. 2020.

10. Shewiyo DH, Kaale E, Risha PG, Dejaegher B, Smeyers-Verbeke J, Vander Heyden Y. Optimization of a reversed-phase-high-performance thin-layer chromatography method for the separation of isoniazid, ethambutol, rifampicin and pyrazinamide in fixed-dose combination antituberculosis tablets. J Chromatogr A. 2012 Oct 19;1260:232-8.

11. Hagga MAM, Sultana S. A novel quantitative method for the simultaneous assay of rifampicin (RIF), isoniazid (INH), ethambutol (EMB), and pyrazinamide (PYP) in 4-FDC tablets. Orient J Chem. 2016;32(6):3081-7.

12. Franco PHC, Chellini PR, Oliveira MAL, Pianetti GA. Simultaneous Determination of First-Line 4-FDC Antituberculosis Drugs by UHPLC-UV and HPLCUV: A Comparative Study. J AOAC Int. 2017 Jan 19;100(4):1008-15.

13. Kurniati Z, Riyanto S, Rohman A. Determination of Rifampicin, Isoniazid, Pyrazinamide and Ethambutol Hydrochloride in 4FDC Tablet by FTIR Spectrophotometry in Combination with Multivariate Calibration. J food Pharm Sci. 2016;4:25-30.

14. Madan J, Dwivedi AK, Singh S. Estimation of antitubercular drugs combination in pharmaceutical formulations using multivariate calibration. Anal Chim Acta. 2005 May 4;538(1-2):345-53.

15. International conference on harmonization of technical requirements for registration of pharmaceuticals for human use (ICH); In: Validation of Analytical Procedures: Text and Methodology, Q2(R1). Geneva; 2005.

16. BRASIL. Agência Nacional de Vigilância Sanitária. RDC No 166, de 24 de Julho de 2017.

17. Akhlaghi SP, Ribeiro IR, Boyd BJ, Loh W. Impact of preparation method and variables on the internal structure, morphology, and presence of liposomes in phytantriol-Pluronic ${ }^{\circledR}$ F127 cubosomes. Colloids Surfaces B Biointerfaces. 2016;145:845-53. 